\title{
Dissipative current in SIFS Josephson junctions
}

\author{
A. S. Vasenko ${ }^{\text {a,b }}$, S. Kawabata ${ }^{\text {c }}$, A. A. Golubov ${ }^{\text {d }}$, M. Yu. Kupriyanov ${ }^{\text {e }}$, F. W. J. Hekking ${ }^{\text {a }}$ \\ ${ }^{a}$ LPMMC, Université Joseph Fourier and CNRS, 25 Avenue des Martyrs, BP 166, 38042 Grenoble, France \\ ${ }^{\mathrm{b}}$ Department of Physics, Moscow State University, Moscow 119992, Russia \\ ${ }^{\mathrm{c} N a n o t e c h n o l o g y}$ Research Institute (NRI), National Institute of Advanced Industrial Science and Technology (AIST), \\ Tsukuba, Ibaraki, 305-8568, Japan \\ ${ }^{\mathrm{d}}$ Faculty of Science and Technology and MESA ${ }^{+}$Institute for Nanotechnology, University of Twente, 7500 AE Enschede, The Netherlands \\ ${ }^{\mathrm{e}}$ Nuclear Physics Institute, Moscow State University, Moscow, 119992, Russia
}

\begin{abstract}
We investigate superconductor/insulator/ferromagnet/superconductor (SIFS) tunnel Josephson junctions in the dirty limit, using the quasiclassical theory. We consider the case of a strong tunnel barrier such that the left $S$ layer and the right FS bilayer are decoupled. We calculate quantitatively the density of states (DOS) in the FS bilayer for arbitrary length of the ferromagnetic layer, using a self-consistent numerical method. We compare these results with a known analytical DOS approximation, which is valid when the ferromagnetic layer is long enough. Finally we calculate quantitatively the current-voltage characteristics of a SIFS junction.
\end{abstract}

Key words: SIFS junction, density of states, dissipative current PACS: 74.45.+c, 74.50.+r, 74.78.Fk, 75.30.Et

\section{Introduction}

It is well known that superconductivity and ferromagnetism are two competing orders. The coexistence of singlet superconductivity and ferromagnetism is basically impossible in the same compound but may be easily achieved in artificially fabricated superconductor/ferromagnet (S/F) hybrid structures. In this case, the coexistence of the two orders is due to the proximity effect $[1,2]$. The main manifestation of the proximity effect in $\mathrm{S} / \mathrm{F}$ structures is the damped oscillatory behavior of superconducting correlations in the $\mathrm{F}$ layer. Two characteristic lengths of the decay and the oscillations are, respectively, $\xi_{f 1}$ and $\xi_{f 2}$.

Therefore in $\mathrm{S} / \mathrm{F}$ heterostructures there is a unique possibility to study the properties of superconducting electrons under the influence of the exchange field in

Email address: Andrey.Vasenko@grenoble.cnrs.fr (A. S. Vasenko). the ferromagnet. Recent progress in the preparation of the high quality S/F layered structures permitted to experimentally observe many striking phenomena that are quite interesting for applications such as a nonmonotonic dependence of their critical temperature and oscillations of critical current in S/F/S Josephson junctions as a function of the $\mathrm{F}$ layer thickness (see [1] and references therein). It is possible to fabricate Josephson $\pi$ junctions with a $\pi$-phase difference in the ground state, which are good candidates for elements in superconducting logic circuits [3].

SIFS junctions, i.e. $\mathrm{S} / \mathrm{F} / \mathrm{S}$ trilayers with one transparent interface and one tunnel barrier between $\mathrm{S}$ and $\mathrm{F}$ layers, represent an interesting case of $\pi$ junctions for applications where active Josephson junctions are required. The SIFS structure offers the freedom to tune the critical current density over a wide range and at the same time to realize high values of the product of the junction critical current $I_{c}$ and its normal state resistance $R_{N}$ [4]. In addition, $\mathrm{Nb}$ based tunnel junctions are 


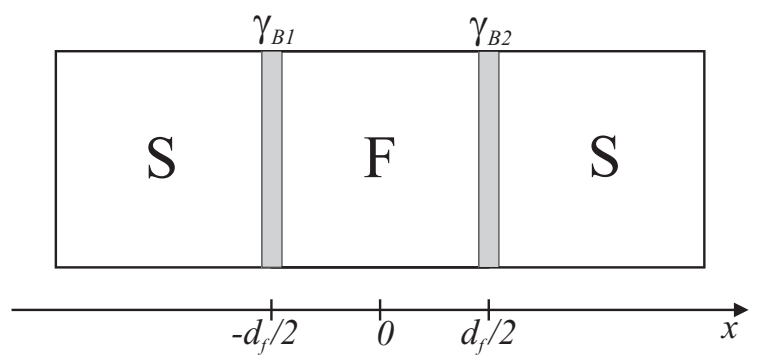

Fig. 1. Geometry of the considered system. The thickness of the ferromagnetic interlayer is $d_{f}$. The transparency of the left $\mathrm{S} / \mathrm{F}$ interface is characterized by the coefficient $\gamma_{B 1}$, and the transparency of the right $\mathrm{F} / \mathrm{S}$ interface is characterized by $\gamma_{B 2}$.

usually underdamped, which is desired for many applications. SIFS junctions are also interesting from the fundamental point of view since they provide a convenient model system for a comparative study between $0-\pi$ transitions observed from the critical current and from the density of states (DOS) [5].

The purpose of this work is to provide a quantitative model describing the DOS in SIFS junctions and to calculate current-voltage characteristics of a SIFS junction. The latter may be used for estimation of the dissipation in SIFS-based qubit systems.

\section{Model and basic equations}

The model of an S/F/S junction we are going to study is depicted in Fig. 1 and consists of a ferromagnetic layer of thickness $d_{f}$ and two thick superconducting electrodes along the $x$ direction. The left superconductor electrode is voltage-biased. Left and right superconductor/ferromagnet interfaces are characterized by the dimensionless parameters $\gamma_{B 1}$ and $\gamma_{B 2}$, respectively, where $\gamma_{B 1, B 2}=R_{B 1, B 2} \sigma_{n} / \xi_{n}, R_{B 1, B 2}$ are the resistances of the left and right $\mathrm{S} / \mathrm{F}$ interfaces, respectively, $\sigma_{n}$ is the conductivity of the F layer, $\xi_{n}=\sqrt{D_{f} / 2 \pi T_{c}}, D_{f}$ is the diffusion coefficient in the ferromagnetic metal and $T_{c}$ is the critical temperature of the superconductor (we assume $\hbar=k_{B}=1$ ). We also assume that the S/F interfaces are not magnetically active. We will consider the diffusive limit, in which the elastic scattering length $\ell$ is much smaller than the characteristic decay length $\xi_{f 1}$.

In this paper we concentrate on the case of a SIFS tunnel Josephson junction, when $\gamma_{B 1} \gg 1$ (tunnel barrier) and $\gamma_{B 2}=0$ (fully transparent interface; however in our numerical calculations we use finite but very small $\left.\gamma_{B 2} \ll 1\right)$. In this case the left $S$ layer and the right FS bilayer in Fig. 1 are decoupled. Therefore we can calculate the quasi-particle current through a SIFS junction using the standard formula of the tunnel theory [6],

$$
I=\frac{1}{e R} \int_{-\infty}^{\infty} d E N_{S}(E-e V) N(E)[f(E-e V)-f(E)],
$$

where $N_{S}(E)=|E| \Theta(|E|-\Delta) / \sqrt{E^{2}-\Delta^{2}}$ is the BCS density of states $[\Theta(x)$ is the Heaviside step function] and $N(E)$ is the density of states in the FS bilayer at the free boundary of the ferromagnet $\left(x=-d_{f} / 2\right)$. Both $N_{S}(E)$ and $N(E)$ are normalized to their values in the normal state; $f(E)=[1+\exp (E / T)]^{-1}$ is the Fermi function. To obtain $N(E)$ we should solve the Usadel equations in the ferromagnetic layer of the FS bilayer.

Using the $\theta$-parameterizations of the normal and anomalous Green functions, $G=\cos \theta, F=\sin \theta$, we can write the Usadel equations in the $F$ layer as $[7,8]$

$$
\frac{D_{f}}{2} \frac{\partial^{2} \theta_{f \uparrow(\downarrow)}}{\partial x^{2}}=\left(\omega \pm i h+\frac{\cos \theta_{f \uparrow(\downarrow)}}{\tau_{m}}\right) \sin \theta_{f \uparrow(\downarrow)},
$$

where a positive (negative) sign in front of $h$ corresponds to the spin up state $\uparrow$ (spin down state $\downarrow), \omega=2 \pi T(n+$ $\left.\frac{1}{2}\right)$ are the Matsubara frequencies, $h$ is the exchange field in the ferromagnet, and the parameter $\tau_{m}$ is the spinflip scattering time. We consider a ferromagnet with strong uniaxial anisotropy, in which case the magnetic scattering does not couple the spin up and spin down electron populations.

In the $\mathrm{S}$ layer the Usadel equations take the form (where we omit subscripts ' $\uparrow(\downarrow)$ ' because equations in superconductor look identically for spin up and spin down electron states),

$$
\frac{D_{s}}{2} \frac{\partial^{2} \theta_{s}}{\partial x^{2}}=\omega \sin \theta_{s}-\Delta(x) \cos \theta_{s} .
$$

They should be completed with the self-consistency equation,

$$
\Delta(x) \ln \frac{T_{c}}{T}=\pi T \sum_{\omega>0}\left(\frac{2 \Delta(x)}{\omega}-\sin \theta_{s \uparrow}-\sin \theta_{s \downarrow}\right) .
$$

Here $D_{s}$ is the diffusion coefficient in the superconductor and $\Delta(x)$ is the superconducting pair potential.

Since we calculate the DOS at the free boundary of the ferromagnet in the FS bilayer, we need to set to zero the $\theta_{f}$ derivative at the left $\mathrm{S} / \mathrm{F}$ interface,

$$
\left(\partial \theta_{f} / \partial x\right)_{-d_{f} / 2}=0
$$

At the right $\mathrm{F} / \mathrm{S}$ interface the boundary conditions are given by the relations [9],

$$
\begin{gathered}
\xi_{n} \gamma\left(\partial \theta_{f} / \partial x\right)_{d_{f} / 2}=\xi_{s}\left(\partial \theta_{s} / \partial x\right)_{d_{f} / 2}, \\
\xi_{n} \gamma_{B 2}\left(\partial \theta_{f} / \partial x\right)_{d_{f} / 2}=\sin \left(\theta_{s}-\theta_{f}\right)_{d_{f} / 2},
\end{gathered}
$$

where $\gamma=\xi_{s} \sigma_{n} / \xi_{n} \sigma_{s}, \sigma_{s}$ is the conductivity of the $\mathrm{S}$ layer and $\xi_{s}=\sqrt{D_{s} / 2 \pi T_{c}}$. The parameter $\gamma$ determines the strength of suppression of superconductivity in the 

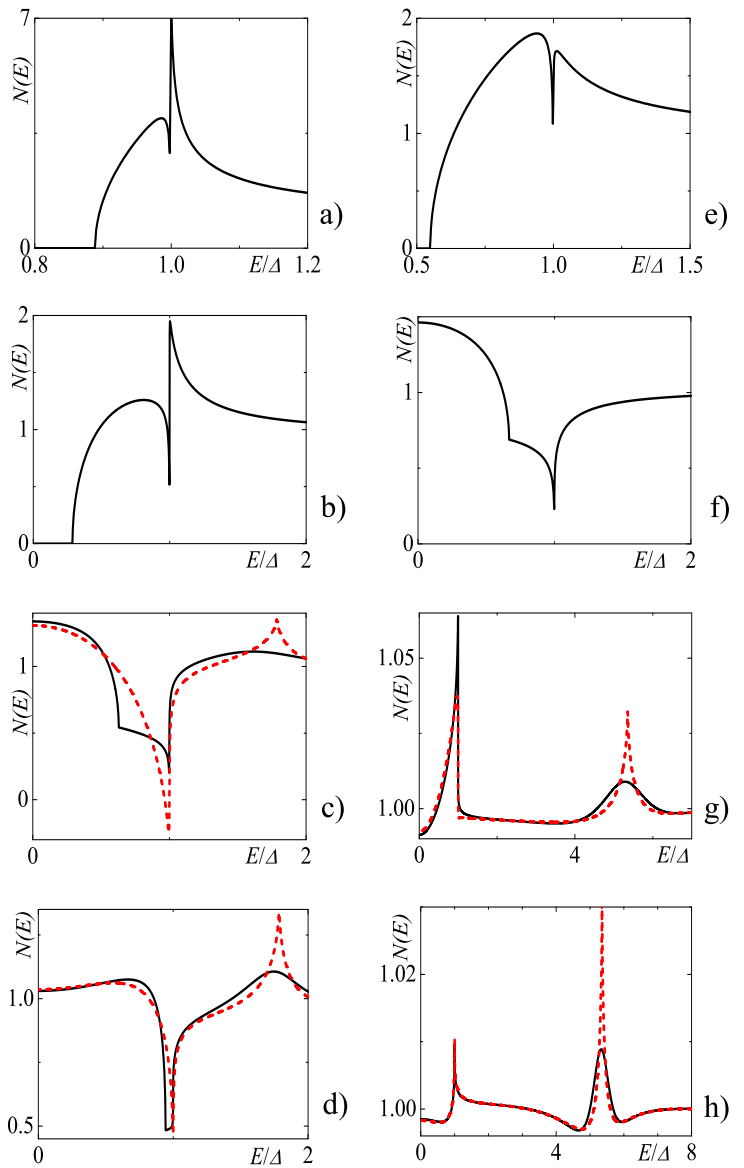

Fig. 2. (Color online) DOS on the free boundary of the F layer in the FS bilayer calculated numerically in the absence of spin-flip scattering. Plots (a)-(d) are calculated for $h=\pi T_{c}$ and (e)-(h) for $h=3 \pi T_{c}$. The temperature $T=0.1 T_{c}$. Parameters of the F/S interface are $\gamma=\gamma_{B 2}=0.01$ (a),(e): $d_{f} / \xi_{n}=0.5$; (b),(f): $d_{f} / \xi_{n}=1$; (c),(g): $d_{f} / \xi_{n}=2$; (d),(h): $d_{f} / \xi_{n}=3$. The approximate analytical solution [5] is shown by dashed red lines.

right $\mathrm{S}$ lead near the interface compared to the bulk: no suppression occurs for $\gamma=0$, while strong suppression takes place for $\gamma \gg 1$. In our numerical calculations we will assume small $\gamma \ll 1$.

To complete the boundary problem we also set a boundary condition at $x=\infty$,

$$
\theta_{s}(\infty)=\arctan \frac{|\Delta|}{\omega}
$$

\section{Density of states in the FS bylayer}

To calculate the DOS $N(E)$ on the free F layer boundary $\left(x=-d_{f} / 2\right)$ we use the self-consistent two-step iterative procedure [10]. In the first step we calculate the pair potential's coordinate dependence $\Delta(x)$ using the self-consistency equation in the S layer, Eq. (4). Then,
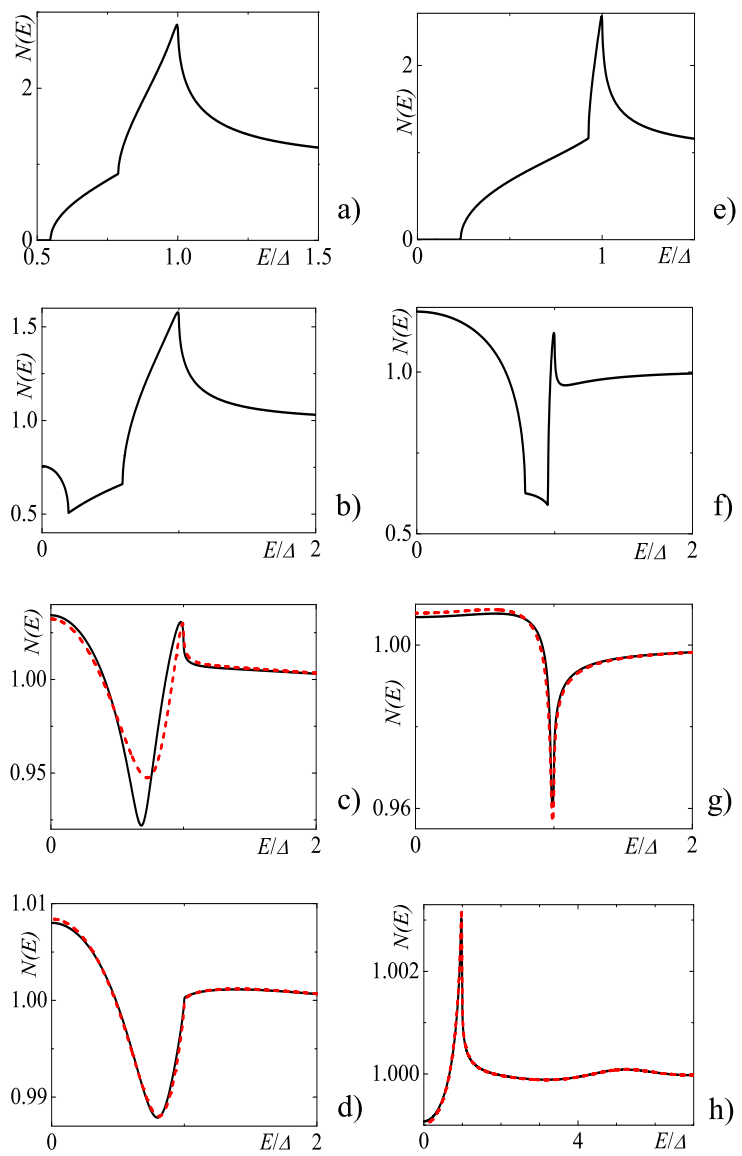

Fig. 3. (Color online) DOS on the free boundary of the F layer in the FS bilayer calculated numerically for $\tau_{m}=1 / \pi T_{c}$. Plots (a)-(d) are calculated for $h=\pi T_{c}$ and (e)-(h) for $h=3 \pi T_{c}$. The temperature $T=0.1 T_{c}$. Parameters of the F/S interface are $\gamma=\gamma_{B 2}=0.01$ (a),(e): $d_{f} / \xi_{n}=0.5$; (b),(f): $d_{f} / \xi_{n}=1$; (c),(g): $d_{f} / \xi_{n}=2 ;(\mathrm{d}),(\mathrm{h}): d_{f} / \xi_{n}=3$. The approximate analytical solution [5] is shown by dashed red lines.

by proceeding to the analytical continuation in Eqs. (2), (3), (5), (6), (7) of the quasi-particle energy $i \omega \rightarrow E+i 0$ and using the $\Delta(x)$ dependence obtained in the previous step, we find the Green functions by repeating the iterations until convergency is reached. We define the full DOS $N(E)$ and the spin resolved DOS $N_{\uparrow(\downarrow)}(E)$, normalized to the DOS in the normal state, as

$$
\begin{aligned}
N(E) & =\left[N_{\uparrow}(E)+N_{\downarrow}(E)\right] / 2, \\
N_{\uparrow(\downarrow)}(E) & =\operatorname{Re}\left[\cos \theta_{b \uparrow(\downarrow)}(i \omega \rightarrow E+i 0)\right],
\end{aligned}
$$

where $\theta_{b}$ is the boundary value of $\theta_{f}$ at $x=-d_{f} / 2$. In case of a long $\mathrm{F}$ layer $\left(d_{f} \gg \xi_{f 1}\right)$ it is also possible to obtain an analytical expression for the DOS at the free boundary of the ferromagnet [5,11].

In Fig. 2 and 3 we plot the DOS energy dependence for different $d_{f}$. Fig. 2 corresponds to the case of the absence of spin-flip scattering (infinite $\tau_{m}$ ) and Fig. 3 

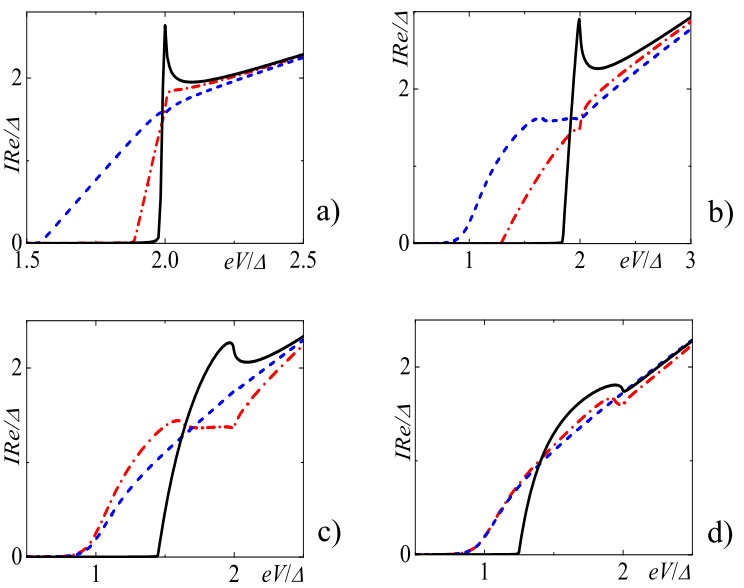

Fig. 4. (Color online) Current-voltage characteristics of a SIFS junction in the absence of spin-flip scattering for different values of the F-layer thickness $d_{f}$. The temperature $T=0.1 T_{c}$. The exchange field $h=0$ (black line, which correspond to the case of a SINS junction), $h=\pi T_{c}$ (red dash-dotted line), and $h=3 \pi T_{c}$ (blue dashed line). (a): $d_{f} / \xi_{n}=0.5$; (b): $d_{f} / \xi_{n}=1$, (c): $d_{f} / \xi_{n}=2$, and (d): $d_{f} / \xi_{n}=3$.

to the case of $\tau_{m}=1 / \pi T_{c}$. In both figures we plot the DOS for two chosen values of exchange field, $h=\pi T_{c}$ for plots (a)-(d) and $h=3 \pi T_{c}$ for plots (e)-(h). In Fig. 2 we see that for any chosen $h$ at small $d_{f}$ the full DOS turns to zero inside a mini-gap, which vanishes with the increase of $d_{f}$. Then the DOS at the Fermi energy $N(0)$ rapidly increases to the values larger than unity and with further increase of $d_{f}$ it oscillates around unity while it's absolute value exponentially approaches unity. In the case of long enough ferromagnets we can observe DOS peaks at $E=h$ [we notice that $\pi T_{c} \approx 1.79 \Delta$ ]. Also for $d_{f} \gtrsim 1.5 \xi_{n}$ [5] we can use an analytical approximation for $N(E)[5,11]$. For smaller $d_{f}$ it is incorrect to use this approximation. It is shown by the dashed red line and is in rather good agreement with the numerical solution. In the numerical curves the peaks at $E=h$ are smeared because we used finite $\gamma=0.01$ for the transparent F/S interface at $x=d_{f} / 2$.

In Fig. 3 we observe similar tendencies. However for smaller $\tau_{m}$ the mini-gap closes at smaller $d_{f}$, the period of the DOS oscillations at the Fermi energy increases and the damped exponential decay occurs faster. Also the DOS peak at $E=h$ is smeared.

\section{Current-voltage characteristics of SIFS}

We calculate the current-voltage characteristics (CVC) of a SIFS junction at $T=0.1 T_{c}$ using Eq. (1). Plots for different parameters are shown in Figs. 4 and 5. For comparison we also present the CVC of a SINS
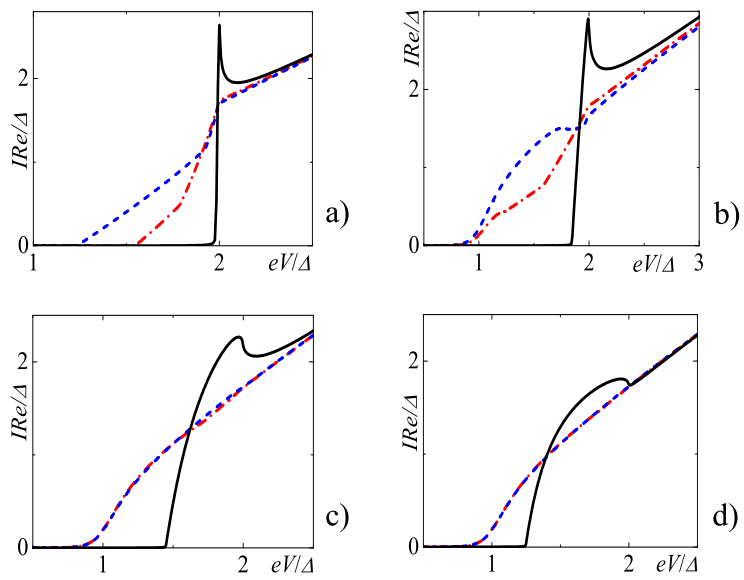

Fig. 5. (Color online) Current-voltage characteristics of a SIFS junction for $\tau_{m}=1 / \pi T_{c}$. The temperature $T=0.1 T_{c}$. The exchange field $h=0$ (black line, which correspond to the case of a SINS junction), $h=\pi T_{c}$ (red dash-dotted line), and $h=3 \pi T_{c}$ (blue dashed line). (a): $d_{f} / \xi_{n}=0.5$; (b): $d_{f} / \xi_{n}=1$, (c): $d_{f} / \xi_{n}=2$, and (d): $d_{f} / \xi_{n}=3$.

tunnel junction, i.e. a junction with a normal metal interlayer instead of a ferromagnet. We can see that for a certain range of parameters the CVC of a SIFS junction exhibits an interesting $\mathcal{N}$-like feature [we can observe it for $h=3 \pi T_{c}$ in Fig. 4 (b) and for $h=\pi T_{c}$ in Fig. 4 (c)]. It corresponds to the case of a large subgap DOS in Fig. 2 (c),(f). For finite spin-flip scattering the features of CVC are smeared. At large $d_{f}$ they totally disappear. A more detailed analysis of CVC of SIFS junctions will be presented elsewhere [12].

\section{Summary}

To summarize, we calculated the quasi-particle DOS in the F layer of a SIFS junction in close vicinity of the tunnel barrier and use it to obtain the current-voltage characteristics of a SIFS junction. The developed formalism may be used for estimations of dissipation in SIFS-based qubits, which will be done elsewhere.

This work was supported by NanoSciERA "Nanofridge" EU project and RFBR Project No. N09-0212176.

\section{References}

[1] A. I. Buzdin, Rev. Mod. Phys. 77, 935 (2005).

[2] A. A. Golubov, M. Yu. Kupriyanov, E. Il'ichev, Rev. Mod. Phys. 76, 411 (2004).

[3] E. Terzioglu and M. R. Beasley, IEEE Trans. Appl. Supercond. 8, 48 (1998); L. B. Ioffe, V. B. Geshkenbein, M. V. Feigel'man, A. L. Fauchère, and G. Blatter, Nature, 398, 679 (1999); 
G. Blatter, V. B. Geshkenbein, L. B. Ioffe, Phys. Rev. B 63, 174511 (2001); A. V. Ustinov and V. K. Kaplunenko, J. Appl. Phys. 94, 5405 (2003); T. Ortlepp, A. Ariando, O. Mielke, C. J. M. Verwijs, K. F. K. Foo, H. Rogalla, F. H. Uhlmann, H. Hilgenkamp, Science, 312, 1495 (2006).

[4] M. Weides, M. Kemmler, E. Goldobin, D. Koelle, R. Kleiner, H. Kohlstedt, and A. Buzdin, Appl. Phys. Lett. 89, 122511 (2006).

[5] A. S. Vasenko, A. A. Golubov, M. Yu. Kupriyanov, and M. Weides, Phys. Rev. B 77, 134507 (2008).

[6] N. R. Werthamer, Phys. Rev. 147, 255 (1966).

[7] K. D. Usadel, Phys. Rev. Lett. 25, 507 (1970).

[8] E. A. Demler, G. B. Arnold and M. R. Beasley, Phys. Rev. B 55, 15174 (1997).

[9] M. Yu. Kuprianov and V. F. Lukichev, Sov. Phys. JETP 67, 1163 (1988) [Zh. Eksp. Teor. Fiz. 94, 139 (1988)].

[10] A. A. Golubov, E. P. Houwman, J. G. Gijsbertsen, V. M. Krasnov, J. Flokstra, H. Rogalla and M. Yu. Kupriyanov, Phys. Rev. B 51, 1073 (1995).

[11] L. Cretinon, A. K. Gupta, H. Sellier, F. Lefloch, M. Faure, A. Buzdin, and H. Courtois, Phys. Rev. B 72, 024511 (2005).

[12] A. S. Vasenko, S. Kawabata, A. A. Golubov, M. Yu. Kupriyanov, and F. W. J. Hekking, in preparation. 